\title{
Philosophiques
}

\section{La théorie des nombres chez Herbrand et Lautman}

\section{Yvon Gauthier}

Volume 37, numéro 1, printemps 2010

Albert Lautman, philosophe des mathématiques

URI : https://id.erudit.org/iderudit/039716ar

DOI : https://doi.org/10.7202/039716ar

Aller au sommaire du numéro

Éditeur(s)

Société de philosophie du Québec

ISSN

0316-2923 (imprimé)

1492-1391 (numérique)

Découvrir la revue

Citer cet article

Gauthier, Y. (2010). La théorie des nombres chez Herbrand et Lautman. Philosophiques, 37(1), 149-161. https://doi.org/10.7202/039716ar

\section{Résumé de l'article}

Dans cet article, je compare les vues de Lautman et Herbrand sur la théorie des nombres et la philosophie de l'arithmétique. Je montre que, bien que Lautman eût avoué avoir été marqué par l'influence de Herbrand, les postures fondationnelles des deux amis divergent considérablement. Alors que Lautman versait dans un réalisme platonicien, Herbrand est resté fidèle au finitisme hilbertien. Il est vrai que Lautman était philosophe et que Herbrand était avant tout arithméticien et logicien, mais il demeure que l'oeuvre de Herbrand a une portée philosophique mieux accordée à la logique et aux mathématiques contemporaines. 


\title{
La théorie des nombres chez Herbrand et Lautman
}

\author{
YVON GAUTHIER \\ Université de Montréal \\ Yvon.gauthier@umontreal.ca
}

\begin{abstract}
RÉSUMÉ. - Dans cet article, je compare les vues de Lautman et Herbrand sur la théorie des nombres et la philosophie de l'arithmétique. Je montre que, bien que Lautman eût avoué avoir été marqué par l'influence de Herbrand, les postures fondationnelles des deux amis divergent considérablement. Alors que Lautman versait dans un réalisme platonicien, Herbrand est resté fidèle au finitisme hilbertien. Il est vrai que Lautman était philosophe et que Herbrand était avant tout arithméticien et logicien, mais il demeure que l'œuvre de Herbrand a une portée philosophique mieux accordée à la logique et aux mathématiques contemporaines.
\end{abstract}

\begin{abstract}
In this paper, I am contrasting Lautman's and Herbrand's views on number theory and philosophy of arithmetic. It is argued that despite the fact that Lautman had acknowledged Herbrand's major influence on his own work, their foundational stances diverge profoundly. Lautman defended a variety of Platonism and Herbrand advocated a personal version of Hilbertian finitism. Of course, Lautman was a philosopher while Herbrand dealt mainly with number theory and logic. It remains though that Herbrand's work is more in tune with contemporary logic and mathematics from a philosophical perspective.
\end{abstract}

\section{Introduction}

Lautman est un fils spirituel de Brunschvicg, le père de la philosophie française des mathématiques, et il a été l'ami de Herbrand - le premier logicien français au sens de la logique mathématique, né aussi en 1908. Brunschvicg s'alliera à Poincaré dans son refus du logicisme des Frege et Russell: son idéalisme, qu'il appellera constructif, est immanent, et le progrès de la conscience qu'il décrira dans Les étapes de la philosophie mathématique de 1912 [5] obéit à une "logique interne » à la démarche mathématique qui demeure réfractaire à la logique formelle. Herbrand, de son côté, optera pour le formalisme, ou plutôt pour le finitisme hilbertien, et s'attaquera au problème de la consistance de l'arithmétique avec un succès partiel, mais sans jamais aller au-delà d'une théorie des démonstrations fidèle à l'esprit hilbertien.

Dans la préface qu'il a écrite pour la réédition des thèses de Lautman, Maurice Loi insiste sur le platonisme de Lautman, mais il y a plus. Outre la thèse principale Essai sur les notions de structure et d'existence en mathématiques [20] et la thèse secondaire Essai sur l'unité des sciences mathématiques dans leur développement actuel [19], on trouve un texte intitulé "Nouvelles recherches sur la structure dialectique des mathématiques ", où assez curieusement Lautman veut mettre à profit certains concepts heideggériens, comme 
l'avait fait Oskar Becker dans son ouvrage de 1927, Mathematische Existenz [3], et comme il l'a fait plus tard dans ses Grundlagen der Mathematik in geschichtlicher Entwicklung de 1954 [4] — Becker va jusqu'à invoquer les niveaux de la réflexion infinie dans la philosophie transcendantale de Schelling pour tenter de justifier les paliers de la hiérarchie des ordinaux transfinis de Cantor. Mais je ne veux pas m'arrêter aux intérêts existentialistes de Lautman, mais plutôt aux soucis logiques qui tiraillent, dit-il, les mathématiques et les modes de liaison des concepts mathématiques. Les dialectiques mathématiques du fini et de l'infini, du discret et du continu, du local et du global, ne sont que des copies d'une réalité idéale qui domine l'activité mathématique ([19] p. 143 et ss.). On trouve alors chez Lautman des expressions comme "montée vers l'absolu", achèvement, perfection d'une figure mathématique qui, si elles témoignent d'un univers transcendant, ne nous instruisent guère sur les mathématiques réelles et leur pratique. Que nous apprend en effet une épistémologie qui nous dit par exemple que la théorie de Galois pour les corps finis (complétions du corps de base K) constitue une montée vers l'absolu ([19] p. 68 et ss.), ou encore que le théorème d'uniformisation pour les courbes algébriques (dû à Poincaré et Koebe) est un achèvement ([19] p. 6 et ss.), ou enfin que c'est une imperfection pour une surface que d'être simplement connexe - un tore serait-il plus parfait qu'un cercle?

Ces analogies ou métaphores ne peuvent être utiles que dans la mesure où elles décrivent l'activité mathématique, elles sont infécondes si elles visent à décalquer le ciel intelligible des idées mathématiques dans la pratique mathématique. À cet égard, la dialectique au sens où l'entend Lautman n'a rien à voir avec la dialectique au sens de Hegel, elle renvoie plutôt à la dialectique platonicienne qui comporte deux mouvements, l'un ascendant

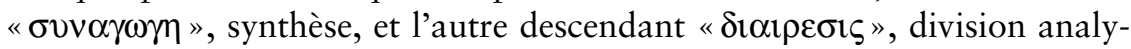
tique, comme l'enseigne le dialogue Parménide de Platon. Lautman dit par exemple:

Il existe ainsi une descente du tout vers la partie comme une montée vers le tout, ce double mouvement s'éclairant à la lumière de l'idée d'achèvement.

Les conditions d'achèvement, comme le note encore Lautman, sont des généralisations conceptuelles qui ont valeur ontologique.

J'ai dit que l'entreprise de Lautman était en bonne partie issue de l'épistémologie historique de Brunschvicg et de son rationalisme idéaliste. Mais Lautman donnera un tour transcendantal ou platonicien à ce rationalisme, et c'est là-dessus que je voudrais insister pour montrer les carences de l'épistémologie lautmanienne en théorie des nombres. 


\section{Logique et théorie des nombres}

Lautman s'inspire de Ingham dans The Distribution of Prime Numbers [15] pour discuter du théorème sur les nombres premiers qui énonce

ou

$$
\operatorname{Lim} \frac{\pi(x)}{x / \log x}=1
$$

$$
\pi(x) \sim x / \log x
$$

qui est une loi asymptotique pour la distribution des nombres premiers avec $\pi(\mathrm{x})$ le nombre de nombres premiers $\leq \mathrm{x}$. Le théorème a fait l'objet de conjectures chez Legendre et Gauss au début du XIX ${ }^{e}$ siècle, et ce n'est qu'en 1896 que Hadamard et de la Vallée Poussin en ont donné une preuve analytique. Ingham lui-même, inspiré par Hardy (et Littlewood), avait supposé que les méthodes transcendantes (de l'analyse complexe ici) étaient inhérentes au problème arithmétique et qu'elles ne sauraient être éliminées. Un autre théorème important en théorie des nombres est le théorème de Dirichlet sur l'infinité des nombres premiers dans toute progression arithmétique $\mathrm{a}+\mathrm{nb}$ où $\mathrm{a}$ et $\mathrm{b}$ sont relativement premiers, c.-à-d. sans dénominateur commun $\neq 1$. Le théorème récent de Green et Tao - lequel vient d'obtenir la médaille Fields pour ses travaux là-dessus - stipule qu'il y a des progressions arithmétiques de longueur arbitraire dans la suite des nombres premiers - le théorème utilise la théorie ergodique et les séries de Fourier, mais on travaille aujourd'hui à en extraire le contenu combinatoire ou constructif, bien qu'il faille entendre constructif dans un sens large puisqu'on admet des méthodes logiques infinitaires (cf. les travaux récents de Avigad, Gerhardy et Townsner [2]). Mais même Terence Tao reconnaît que la méthode de Herbrand pourrait permettre de constructiviser encore davantage les résultats analytiques.

Voici en bref la formulation de Herbrand. Soit $A$ une formule en forme prenexe, par exemple

$$
A \equiv \exists x \forall y \exists z \forall t R(x, y, z, t)
$$

avec $R$ sans quantificateur. On introduit deux nouvelles lettres de fonctions, $f$ unaire et $g$ binaire avec les termes $U_{1} \ldots U_{n}, W_{1} \ldots W_{n}$, alors est démontrable dans le calcul des prédicats sous la forme

$$
A \equiv B\left(U_{1}, f\left(U_{1}\right), W_{1}, g\left(U_{1}, W_{1}\right)\right) \vee \ldots \vee B\left(U_{n}, f\left(U_{n}\right), W_{n}, g\left(U_{n}, W_{n}\right)\right) .
$$

Cette disjonction est dérivable dans un calcul propositionnel et peut servir de critère de réfutabilité dans une interprétation négative (voir Hilbert et Bernays [14], II, pp. 170 et ss).

La négation de $A$ est

$$
\neg A \equiv \forall x \exists y \forall z \exists t \neg B(x, y, z, t)
$$


ou

$\neg A \equiv \neg B(x, f(x), z, g(x, y))$

et si Herbrand a vu la consistance dans la réfutabilité sur un «champ infini » ou indéfini, Kreisel a pensé l'interprétation sans contre-exemple comme une interprétation fonctionnelle sur les types supérieurs; les fonctionnelles récursives sont de la forme

$$
B x_{1} \ldots x_{n}\left[F_{1}\left(f_{1}, \ldots, f_{n}\right), \ldots, F_{m}\left(F_{1}, \ldots, F_{n}\right)\right]
$$

avec $B$ ouverte. Pour une formule vraie $A$, nous avons

$$
B[F(f, g), f(F(f, g)), G(F(f, g), G(F, g))]
$$

où les $F$ et les $G$ sont évidemment nos nouvelles fonctionnelles récursives sur les types. forme

La dernière formule $A$ est vraie s'il n'y a pas de contre-exemple de la

$$
\neg B[x, f(x), z, g(x, y)]
$$

avec $f$ et $g$ comme arguments des fonctionnelles récursives $F$ et $G$ de type supérieur; $F$ et $G$ sont continues et peuvent donc être associées à des polynômes de degré arbitraire: nous pouvons définir la composition de $F$ et $G$

$$
F \bullet G=\left(\sum_{i} F_{i} x^{i}\right)\left(\sum_{j} G_{j} x^{j}\right) \sum_{i} \sum_{j}\left(F_{i} G_{i} x^{i+j}\right) .
$$

Puisque nous ne pouvons quantifier sur toutes les fonctionnelles - par diagonalisation il y a une fonctionnelle récursive qui est distincte de toutes les fonctionnelles récursives - nous devons nous restreindre aux polynômes de degré fini et utiliser la descente sur les degrés et les hauteurs de polynômes pour retrouver une version finitiste (voir là-dessus [13] pour cette construction).

Remarquons que les fonctions récursives primitives peuvent se traduire aisément en fonctions polynomiales. La chose est évidente pour les fonctions constantes initiales; la composition et la récursion sont traitées comme un produit de convolution $G \bullet H$ pour $G$ et $H$ de telle sorte que

$$
\begin{aligned}
& F(x)_{\vec{n}}=G_{n}\left(H_{1}\left(a_{n}\right), \ldots, H_{p}\left(a_{n}\right)\right) \\
& \text { avec } H \bullet G=\sum_{i} \sum_{i}\left(G_{i} H_{i} x^{i+j}\right) .
\end{aligned}
$$

L'opérateur comme l'équivalent au principe du plus petit nombre est remplacé par la descente (finie) infinie sur les puissances décroissantes d'un polynôme de degré fini

$$
F(x)_{\bar{n}}=f_{0} x^{n}+f_{1} x^{n-1}+\ldots+f_{n-1} x+f_{n} .
$$


Selon l'idée de Hilbert d'une suite terminale de prédécesseurs pour un $n$ donné, la descente fermatienne autorise un processus de réduction fini à la façon d'un ordre linéaire décroissant de puissances pour un polynôme donné.

Les deux théorèmes sur la distribution asymptotique des nombres premiers et le théorème de Dirichlet sur l'infinité des nombres premiers dans toute progression arithmétique, on le sait maintenant, ont des versions élémentaires obtenues par Selberg et Erdös en 1949 et en 1950. Élémentaire signifie ici que l'on n'utilise que des méthodes arithmétiques constructives ou combinatoires comme les sommes finies, sans recours aux méthodes transcendantes comme le prolongement analytique de séries infinies en analyse complexe.

Je ferai remarquer que Dirichlet, dans son texte de 1836, avouait que:

Es fehlt noch an gehörigen Principien, unter denen transzendente Verbindungen, welche unbestimmte ganze Zablen enthalten, verschwinden können (Werke I [21], p. 326).

notant donc qu'il manque encore les principes appropriés en vertu desquels les relations transcendantes (obtenues par les séries infinies) entre des entiers indéterminés pourraient être éliminées. Ce n'est donc pas sans réticence que Dirichlet avait introduit la méthode analytique des séries infinies en théorie des nombres. Kronecker, qui a édité les Werke de Dirichlet, a proposé dans ses Vorlesungen über Zablentheorie [17] d'étendre arithmétiquement un intervalle fini $(\mu \ldots v)$ pour les entiers $\mu$ et $v$ afin d'y loger au moins un nombre premier $\mathrm{hm}+\mathrm{r}$ pour $\mathrm{m}$ et $\mathrm{r}$ relativement premiers. On pourrait voir là une anticipation des idées de Selberg, qui utilise des formules asymptotiques pour la fonction logarithmique sur des segments ou intervalles finis de $Z-$ voir de nouveau [11] p. 36.

Pour Lautman, dans le cas du théorème sur les nombres premiers associé à l'hypothèse de Riemann pour les zéros de la fonction $\zeta(\mathrm{s})$ sur la droite réelle $1 / 2 \ldots$, il s'agit de la dialectique du continu (analyse) et du discontinu (arithmétique) ([19] p. 233). Je noterai encore que l'hypothèse de Riemann est née d'une remarque marginale dans le texte de 1859 « Über die Anzabl der Primzahlen unter einer gegebenen Grösse» [22], et que Riemann l'a laissée de côté «bei Seite gelassen " puisqu'il s'intéressait d'abord au problème de la distribution des nombres premiers. Cette remarque en appelle une autre, aussi marginale, de Fermat sur son "dernier» théorème! Dans son exposé, Lautman privilégie l'analyse et va jusqu'à dire que "l'impossibilité d'éliminer l'analyse de l'arithmétique » ([19] p. 214) dans le théorème sur les nombres premiers est comparable au théorème de Gödel sur les preuves de non-contradiction (deuxième théorème d'incomplétude de Gödel) : l'histoire lui a donné tort là-dessus, et il est facile d'expliquer pour quelle raison. L'absence d'un point de vue fondationnel ne lui a pas permis 
de voir que le véritable enjeu se situait au niveau des méthodes de preuve, comme l'avait souligné l'intuitionnisme et comme l'avait reconnu Herbrand. Dans ses travaux sur la consistance de l'arithmétique, Herbrand adopte le point de vue finitiste de Hilbert. Herbrand, par-delà Hilbert, revient à la posture fondationnelle de Kronecker, et ce qu'il appelle argument intuitionniste est en fait une procédure constructive quand il déclare qu'il ne suppose pas qu'un objet existe sans qu'on puisse trouver un moyen de le construire; de même ce qu'il appelle un champ infini n'est qu'une abréviation pour la construction itérative du $<$ pas à pas $>(<$ Schritt $z u$ Schritt $>)$ d'un champ ou domaine d'objets illimité ou "effini", comme je préfère le dire. Son théorème fondamental porte sur l'identité $a=a$ ou la non-identité $a \neq a$, ce que Hilbert désignait par équation ou inéquation. Si $P$ est une identité, une preuve de $P$ va nous permettre de trouver un nombre $b$ tel que $\neg P$ ne puisse être vraie dans tout domaine d'ordre $h$; de même, si $P$ n'est pas une identité, pour tout $h$ on peut construire un domaine d'ordre $h$ dans lequel $\neg P$ est vraie. L'idée de Herbrand dans sa preuve de consistance est de se servir d'une induction sans quantificateurs (sans variables liées) et de laisser libre cours à des fonctions récursives générales obtenues par substitution et récursion. Herbrand est bien conscient qu'il n'y a pas de procédure générale ou d'algorithme pour définir toutes les fonctions récursives et que c'est la seule méthode diagonale qui permet à Gödel de passer outre pour démontrer l'incomplétude de l'arithmétique. L'arithmétique récursive de Herbrand peut elle aussi se traduire facilement en termes de polynômes où l'on remplace la notion d'ordre d'un domaine par celle de degré, et il saute aux yeux qu'un polynôme de degré fini obtenu par composition récursive (substitution et récursion) est soumis à la diagonale de Cauchy (ou produit de convolution) et échappe à la diagonale de Cantor qui suppose une quantification sur l'ensemble des nombres naturels, ce que refuse Herbrand dans sa démarche intuitionniste ou constructiviste. C'est cependant dans sa thèse de 1930 qu'il formule son théorème sur la consistance du calcul des prédicats importants aussi en informatique théorique, comme je l'ai annoncé plus haut. Je ne reviendrai pas là-dessus, si ce n'est que pour insister sur le fait que le programme de Herbrand, si on peut l'appeler ainsi, a donné naissance à l'interprétation sans contre-exemple de Kreisel (à partir de l'exposé de Hilbert et Bernays dans Grundlagen der Mathematik) et à son programme du < proof mining $>$ ou extraction du minerai constructif des théorèmes classiques de l'analyse. Programme qui est relancé aujourd'hui surtout par Ulrich Kohlenbach à Darmstadt. Ce qui importe à nos yeux dans la démarche de Herbrand, c'est son insistance sur les méthodes finitaires et le point de vue fondationnel qui s'y rattache. Ainsi, Herbrand formulera l'hypothèse suivante, que j'ai appelée il y a plusieurs années la conjecture de Herbrand:

Les méthodes transcendantes ne peuvent permettre de démontrer en arithmétique des théorèmes qu'on ne puisse démontrer sans leur aide (Écrits logiques [12] p. 152). 
Dans un article récent «Number Theory and Elementary Arithmetic» [1], Jeremy Avigad, sans mentionner Herbrand, attribue à Harvey Friedman la conjecture suivante, qu'il appelle Grand Conjecture:

Every theorem published in the Annals of Mathematics whose statement involves only finitary mathematical objects (i.e. what logicians call an arithmetical statement) can be proved in elementary arithmetic.

Cela inclut évidemment le dernier théorème de Fermat dont la preuve par Andrew Wiles a occupé tout un numéro des Annals of Mathematics en 1995: il faudrait donc dégager cette preuve de sa gangue analytique! Le logicien contemporain entend par élémentaire un sous-système de l'arithmétique de Peano du premier ordre ou encore l'arithmétique récursive primitive avec induction bornée. Pour ma part, je soutiens que même l'arithmétique récursive primitive ne capture pas l'arithmétique classique, et j'ai proposé dans mes travaux de substituer la descente infinie de Fermat à l'induction complète que traduit le postulat d'induction de Peano en montrant que les deux principes ne sont pas équivalents du point de vue constructiviste, ce que même les intuitionnistes n'ont pas vu puisqu'ils identifient la descente infinie à l'induction transfinie, et donc à l'induction complète sur les ordinaux de la deuxième classe de nombres de Cantor

$$
\lim \omega=\varepsilon_{0}
$$

mais il y a là une double négation sur l'ensemble infini des nombres naturels $N$. La conjecture de Herbrand échappe donc à Lautman, et Dieudonné a mal évalué les dons de prophétie de Lautman, comme il le prétendait dans l'avant-propos critique de l'Essai sur l'unité des sciences mathématiques [19]. On pourrait d'ailleurs minimiser la comparaison que Lautman tente d'établir avec le deuxième théorème d'incomplétude de Gödel ou théorème sur les preuves de consistance, puisque Gödel lui-même n'écarte pas la possibilité d'une preuve finitaire de la consistance de l'arithmétique dans une remarque de son texte de 1931 sur laquelle il reviendra plus tard — on peut penser qu'il a tenté de formuler une telle preuve dans son interprétation Dialectica «Über eine noch nicht benützte Erweiterung des finiten Standpunktes» où l'on a des fonctionnelles de type fini au lieu de l'induction transfinie de Gentzen et Ackermann.

Mais on ne doit pas jeter le bébé avec l'eau du bain, disait déjà Riemann «das Kind mit dem Bade ausschütten» dans un autre contexte - il s'agissait de l'a priori formel kantien qu'il voulait remplacer par un a priori matériel, comme son maître en philosophie Johann Friedrich Herbart l'enseignait. Si on ne peut nier l'utilité des méthodes analytiques, tous les mathématiciens s'entendent pour accorder un statut particulier aux preuves constructives directes, "effectives» qui donnent plus d'information que les méthodes transcendantes souvent plus élégantes, parce qu'elles empruntent un détour via les objets idéaux, dirait Hilbert. C'est en réalité une théorie 
des preuves, constructives ou autres, qui aura manqué à l'entreprise de Lautman.

\section{Dialectique et théorie des nombres algébriques}

Sa dialectique des genèses et des structures, Lautman aurait voulu l'enrichir par une théorie des mixtes au sens platonicien des genres composés, dyades ou mélanges d'essence et d'existence, ou encore par un principe de dualité entre le continu et le discontinu. Je voudrais donner un autre exemple, cette fois en théorie des nombres algébriques, et montrer comment la dialectique des concepts est à certains égards superficielle ou artificielle. Il s'agit de la distinction entre corps de nombres algébriques et corps de fonctions algébriques que Lautman voudrait dissocier dans la dualité du continu et du discontinu.

Prenons $Q[x]$ l'anneau des polynômes en une indéterminée avec coefficients dans le corps $Q$ des nombres rationnels. Un corps de fonctions algébriques à une variable dans $Q$ est une extension de degré fini de $Q[x]$. Les corps de fonctions algébriques définis sur $Q[x]$ sont les analogues "parfaits» des corps de nombres algébriques définis sur l'anneau des entiers $Z$. Le sous-corps des quotients $Q(x)$ de $Q[x]$ est par exemple le corps des fonctions rationnelles avec coefficients dans $Q$. La géométrie algébrique récente fait son pain quotidien de ces sous-corps. La théorie des schémas de Grothendieck, par exemple, c'est Dieudonné lui-même qui l'a reconnu, est la descendante de la théorie des domaines de rationalité "Rationalitätsbereiche» de Kronecker, qui, soit dit en passant, préférait ce terme à celui de corps «Körper» employé par Dedekind et qu'il trouvait trop chargé de matière - ce qui aurait sans doute plu à l'idéaliste Lautman s'il avait connu Kronecker et sa théorie des formes ou polynômes homogènes. Kronecker se contentait des extensions simples par l'adjonction d'un seul élément à la fois, alors que l'on a maintenant des extensions infinies, algébriques ou transcendantes, celles-ci étant adjointes en bloc d'ensembles, peut-on dire.

Je remarquerai en plus que la géométrie algébrique que l'on renomme aujourd'hui géométrie arithmétique a pris acte de l'intime connexion des deux corps, le corps des nombres algébriques et le corps des fonctions algébriques, dans le programme de Langlands notamment. Langlands s'inspire directement du < Jugendtraum > de Kronecker (voir Langlands [18]), pour qui l'analyse était le point de départ et l'algèbre le moyen d'atteindre la cible qui est l'arithmétique. Pour Kronecker, c'est bien l'arithmétisation de l'algèbre qui est la fin ultime. Pour le programme motivique ou théorie des correspondances algébriques de Grothendieck, on le considère encore trop spéculatif, mais ce sont les résultats de finitude (de points rationnels sur les courbes elliptiques) de Faltings sur la conjecture de Mordell et de Deligne sur la conjecture de Weil pour l'hypothèse de Riemann sur les variétés rationnelles qui ont été le point focal de l'attention dans cette direction en géométrie arithmétique contemporaine. Il y a là un fil directeur: Poincaré a 
étudié d'abord les propriétés arithmétiques des courbes algébriques en utilisant une version de la descente infinie dans l'expression "un nombre fini d'hypothèses ", Mordell a ensuite démontré la conjecture de Poincaré sur la génération finie des points rationnels sur une courbe elliptique en définissant la descente infinie comme méthode partant d'un entier $n$ donné pour descendre finiment; enfin la conjecture de Mordell sur le nombre fini de points rationnels sur une courbe elliptique de genre $>1$ a été démontrée par Faltings qui a eu recours à une forme de descente généralisée. Quant à André Weil, qui a joué un rôle majeur dans toute cette histoire, on sait que sa méthode de preuve privilégiée était «la descente finie dans les corps finis». Récemment le mathématicien français Laurent Lafforgue, médaillé Fields, a réussi à montrer la correspondance exacte de Langlands entre morceaux de l'espace modulaire (ou variété algébrique) et points rationnels (dénombrables) à l'aide d'une technique d'itération des chtoukas de Drinfeld «штука» du russe vient de l'allemand «Stücke», morceaux. Le corps de base de la correspondance de Langlands est un corps fini $\mathrm{F}$ avec groupe de Galois G. Ici, Lautman parlerait plutôt d'imperfection du corps de base ([19] p. 65) et dirait qu'il faut engendrer un sur-corps par montée vers l'achèvement, alors qu'il ne s'agit que d'une extension du corps de base, et le corps $Q$ des rationnels n'est qu'un sous-corps du corps $R$ des réels, luimême sous-corps de $C$ des nombres complexes. Mais ici, dans la théorie des corps finis, la descente infinie de Fermat s'applique parfaitement, comme l'a bien montré André Weil dans son ouvrage Number Theory. An approach through history. From Hammourabi to Legendre [23], et comme il l'a fait dans sa pratique ${ }^{1}$. Ce que Lautman ne pouvait voir évidemment. Ni Cavaillès d'ailleurs, qui assez naïvement disait que toute théorie contenant l'arithmétique des entiers tombait sous le coup de l'incomplétude de Gödel, et il ajoutait que c'est à peu près toute théorie mathématique qui était ainsi atteinte - voir Sur la logique et la théorie de la science ([6] p. 70). Lautman eût-il caractérisé la descente infinie comme descente vers l'imparfait s'il l'avait connue? Et la théorie du corps de classes d'idéaux qui relève de la

1. Je pourrais faire remarquer ici que les théoriciens des modèles contemporains s'inspirent largement de la théorie des corps finis, comme le notait Ehud Hrushovski dans une conférence plénière du dernier congrès de Logique, méthodologie et philosophie des sciences à Beijing en août 2007. La conférence de clôture de Hrushovski portait sur l'héritage de Gödel, et Hrushovski a insisté avec justesse sur le fait que le théorème de complétude était plus important pour la théorie des modèles que les résultats d'incomplétude; pour lui, les résultats d'André Weil en théorie des corps finis jouaient un rôle encore plus important en théorie contemporaine des modèles. Là-dessus, je lui ai fait remarquer que justement l'outil privilégié de Weil en théorie des corps finis était la méthode de la descente infinie (en réalité finie) de Fermat et que Weil n'admettait pas la méthode diagonale de Cantor comme méthode de preuve valide en théorie des nombres, et on sait qu'elle est cruciale dans le premier théorème d'incomplétude de Gödel ; ce que Hrushovski aurait dû relever, comme je le lui ai suggéré. J’ai ajouté que le phénomène d'incomplétude affectait moins de $20 \%$ des mathématiques actuelles... ce qu'il a reconnu aussi. 
théorie algébrique des nombres et que Lautman a abordée après que Herbrand l'eut pratiquée - avec des méthodes arithmétiques - repose sur l'idée que le nombre de classes d'idéaux est fini...

Les quelques résultats de Herbrand en théorie des nombres sont classiques: ils établissent une relation entre les nombres de Bernoulli, valeurs numériques des coefficients $\mathrm{x}^{2} / 2$ ! $\mathrm{x}^{4} / 4$ !, $\ldots$, dans l'expansion de $\mathrm{x} /\left(1-\mathrm{e}^{-\mathrm{x}}\right)$, avec la structure de groupe des classes d'idéaux de $Q\left(\zeta_{p}\right)$ pour la fonction $\zeta$ sur les nombres premiers - voir K. Ireland et M. Rosen, A classical Introduction to Number Theory [16]. D'ailleurs, Herbrand a aussi travaillé à la théorie des corps cyclotomiques qu'il appelle circulaires en s'inspirant du $12^{\mathrm{e}}$ problème de Hilbert sur "L'extension de la proposition de Kronecker sur les corps abéliens dans un domaine de rationalité algébrique »; le théorème de Kronecker-Weber stipule que toute extension abélienne de $Q$ appartient au corps cyclotomique des racines de l'unité, comme l'avait décrit Kummer - cf. l'ouvrage de Herbrand Le développement moderne de la théorie des corps algébriques. Corps de classe et lois de réciprocité, [13] publié en 1936 par son ami Claude Chevalley. Dans ce contexte, la dialectique de Lautman est sinueuse, puisqu'il évoque en les approuvant ([19] p. 189) les résultats arithmétiques pour la fonction $\zeta$ sur les corps finis (de nombres rationnels); il reconnaît aussi que les mathématiques classiques avant Cantor sont d'essence constructiviste! Mais il ne faut pas accabler Lautman là-dessus. Même des philosophes des mathématiques contemporains comme David Corfield, dans son essai récent Towards a Philosophy of Real Mathematics [8], lequel parle de "disanalogy» ou fausse analogie entre corps de nombres et corps de fonctions, alors que le mathématicien parle d'analogie profonde ou de correspondance exacte ou encore d'une théorie unifiée comme Harold Edwards dans sa Divisor Theory [9]. Enfin, je pourrais ajouter ici avec un brin d'ironie que la distinction entre géomètres et arithméticiens tient (peut-être) au fait que les géomètres n'ont lu que la première partie des Livres d'Euclide sur la géométrie, alors que les arithméticiens les ont lus jusqu'au bout en incluant les Livres arithmétiques. Et les arithméticiens, ce sont eux qui font aujourd'hui de la géométrie arithmétique!

\section{Conclusion}

Je voudrais terminer en commentant quelques remarques de Lautman sur la logique. Je cite Lautman ([19] p. 39):

La véritable logique n'est pas a priori par rapport aux mathématiques, mais il faut à la logique une mathématique pour exister.

Il ajoutait que si la logique n'admet pas les définitions imprédicatives, les mathématiques s'en nourrissent. Cette conception brunschvicgienne et poincaréenne, nous la partageons, et elle est commune chez les mathématiciens, mais elle ne justifie pas pour autant les preuves d'existence, véritables 
preuves ontologiques de l'existence des êtres mathématiques dans l'esprit de Lautman. Pour Lautman, l'idée de parfait précède l'idée d'imparfait, comme chez Descartes qui pensait que l'idée d'infini ne pouvait être injectée dans un esprit fini que par un être infini - mais Descartes, comme Leibniz, comme Gauss, comme Cauchy, rejetait l'idée d'infini mathématique. La logique ou la dialectique interne des constructions mathématiques n'a pas besoin de recourir aux «relations logiques entre l'essence et l'existence» ([19] p. 80) pour rendre compte de l'objectivité des mathématiques. Poincaré, avant Herbrand, dans ses Dernières Pensées pensait que l'infini n'est qu'une abréviation du fini, c'est-à-dire que l'infini est une approximation du fini, une idée reprise par Y. Gurevitch, un des créateurs de la théorie contemporaine des modèles finis. Si Lautman parle de l'indépendance de la logique dialectique - au sens où il l'entend — et des mathématiques ([19] p. 139), c'est qu'il se fonde sur un platonisme des relations entre les théories mathématiques et le ciel d'idéalités qui les domine. Lautman évoque volontiers la nature imparfaite de certains êtres mathématiques plutôt que des constructions d'objets déficientes. Il dit de la théorie des démonstrations que c'est une théorie structurale alors que le point de vue ensembliste est extensif; pour lui la notion de réalisation dans un champ infini, en réalité potentiellement infini, chez Herbrand est la création d'êtres mathématiques hybrides ou mixtes entre deux genres de l'être ([19] chap. III).

Je n'insisterai pas davantage sur ces remarques disparates de Lautman, si ce n'est pour conclure que si sa culture logique était mince, sa culture mathématique en menait large, trop large peut-être pour ne pas demeurer superficielle dans bien des cas. Quoi qu'il en soit, le réalisme platonicien de Lautman est impuissant à définir une option fondationnelle. Si Lautman possédait sans doute parmi les philosophes la plus vaste érudition mathématique, Cavaillès, qui n'était pas aussi savant, était cependant un philosophe plus pénétrant. Cavaillès tente de penser plus concrètement «le mouvement des liaisons intellectuelles dans leur développement dialectique", selon l'expression de Lautman; c'est le lien entre la superposition intuitive et la dialectique des concepts qui reste le problème fondamental de la philosophie mathématique, écrit Cavaillès dans sa Philosophie mathématique ([7] p. 273). Il entendait par superposition intuitive une sorte de méta-intuition, comme on dit métalangage, qui s'appuie sur une intuition première, laquelle est toujours en procès de dissolution. Qu'entendait-il par dialectique du concept? Il refusait la primauté de la conscience transcendantale dans la théorie husserlienne sur l'enchaînement dialectique des concepts. L'interaction des gestes constructeurs et des objets construits n'est pas définie une fois pour toutes, elle est la reprise indéfinie du jeu dialectique dans une histoire dont la seule nécessité est interne. On pourrait reprocher à Cavaillès d'avoir trop peu exemplifié son propos: l'histoire de la théorie des ensembles qu'il a surtout privilégiée n'est qu'un petit chapitre dans l'histoire des mathématiques. Il n'avait pas le vaste horizon de Lautman. Le mérite de 
Cavaillès est d'avoir indiqué que les idéalités — les constructions - mathématiques jouissent de l'autonomie d'un texte continu unifié par le concours d'actes dont on peut dire qu'ils convergent uniformément si l'on tient compte de la continuité historique des théories mathématiques. Il n'y pas d'histoire mieux enchaînée, plus liée que l'histoire des mathématiques, et pourtant, à chacun de ses moments, elle n'est que la suite aléatoire des concepts nouveaux, répétition du même dans l'autre et reduplication des possibles dans un langage neuf.

Un Desanti qui s'inspire de Cavaillès et de Husserl n'a pas su retenir tous les éléments de cette synthèse presque brunschvicgienne de l'intuitif et de l'axiomatique (Brunschvicg disait vérification), synthèse que la logique mathématique contemporaine a retrouvée par d'autres moyens - voir làdessus mon article "L'épistémologie française des mathématiques », dans la revue Critique, paru en 1979 ([10], pp. 3-36). C'est l'égologie transcendantale de Husserl qui est mise entre parenthèses chez Desanti comme chez Cavaillès, mais ce sont les idéalités mathématiques comme chez Lautman que Desanti veut réanimer, cette fois dans la dialectique objective d'une procession abstraite des concepts. Mais Desanti perpétue en quelque sorte la méfiance à l'égard de la logique héritée de Poincaré et de Brunschvicg, relayée par Lautman et Cavaillès, et il faudra attendre les travaux de Lacombe et Fraissé, puis ceux de Poizat et Girard parmi d'autres pour reprendre la logique mathématique là où l'avait laissée Herbrand en France. Seul Herbrand aura eu une descendance, et son ami Lautman n'aura pas eu d'héritiers d'obédience platonicienne en philosophie mathématique ou en fondements des mathématiques, ou encore en recherche fondationnelle, comme on doit dire maintenant ${ }^{2}$.

\section{Références bibliographiques}

Avigad, J. «Number Theory and Elementary Arithmetic ", Philosophia Mathematica XI, 2003, pp. 257-284.

Avigad, J., P. Gerhardy et A. Townsner. «Local Stability of Ergodic Averages ", à paraître dans Trans. of A.M.S.

2. P.S. Sur une note personnelle. Brunschvicg a été la première influence et peut-être la plus durable - j'ai rédigé un mémoire de maîtrise sur Brunschvicg au département de philosophie de l'Université de Montréal il y a près d'un demi-siècle! L. Brunschvicg avait déjà utilisé dans son ouvrage Les étapes de la philosophie mathématique de 1912 les termes d'arithmétisme - et de logique interne, mais dans un sens différent du mien — que j'ai réintroduit dans un ouvrage récent sur l'arithmétisation de la logique. Pour lui, l'arithmétisme ou le pythagorisme s'opposait à ce qu'il appelait à l'époque logistique - il emploie même le terme < logisticisme > pour logicisme - , mais c'était un dogme rigide qui n'épousait pas la dialectique interne de la création mathématique, une terminologie qui évoque cette fois la longue lignée des héritiers de Brunschvicg, Lautman, Cavaillès ou Desanti qui, en se détachant de Husserl pour les deux derniers, ne s'éloignent pas pour autant de Brunschvicg et de son constructivisme ou idéalisme immanent, dont je me réclame aussi librement... 
Becker, O. "Mathematische Existenz », Jahrbuch für Philosophie und phänomenologische Forschung IX, 1927.

- Grundlagen der Mathematik in geschichtlicher Entwiclung, Alber, Freiburg/ München, 1954.

Brunschvicg, L. Les étapes de la philosophie mathématique, PUF, Paris, 1947.

Cavaillès, J. Sur la logique et la théorie de la science, Vrin, Paris, 1976.

—. Philosophie mathématique, Hermann, Paris, 1962.

Corfield, D. Towards a philosophy of real mathematics, Cambridge University Press, Cambridge, 2006.

Edwards, H. Divisor Theory, Birkhaüser, Basel, 1990.

Gauthier, Y. «L'épistémologie française des mathématiques », Critique, Paris, 1979, pp. 3-36.

- Internal Logic. Foundations of Mathematics from Kronecker to Hilbert, Kluwer «Synthese Library ", Dordrecht/Boston/London, 2002.

Herbrand, J. Écrits logiques, PUF, Paris, 1968.

- Le développement moderne de la théorie des corps algébriques. Corps de classe et lois de réciprocité, Gauthier-Villars, Paris, 1936.

Hilbert, D. et P. Bernays. Grundlagen der Mathematik I et II, 2 Aufl., Berlin, SpringerVerlag, 1968 et 1970.

Ingham, A. E. The Distribution of Prime Numbers, Cambridge University Press, Cambridge, 1932.

Ireland, K. et M. Rosen. A Classical Introduction to Modern Number Theory, Berlin, Springer-Verlag, 1980.

Kronecker, L. Vorlesungen über Zablentheorie, vol. I, K. Hensel (ed.), Teubner, Leipzig, 1901.

Langlands, R.P., "Some Contemporary Problems with Origins in the Jugendtraum », Mathematical developments arising from Hilbert's problems, American Mathematical Society, Providence, R.I., 1976.

Lautman, A. Essai sur l'unité des sciences mathématiques dans leur développement actuel, Hermann, Paris, 1938.

_. Essai sur les notions de structure et d'existence en mathématiques, Hermann, Paris, 1938.

Lejeune Dirichlet, P.G. Werke I, hrsg. v. L. Kronecker, Reimer, Berlin, 1889.

Riemann, B. Gesammelte mathematische Werke, wissenschaftlicher Nachlass und Nachträge. Collected Papers neu hrsg. v. R. Naramsihan, (B.G Teubner, Springer-Verlag, Berlin, New York, Leipzig), 1990.

Weil, A. Number Theory. An Approach Through History. From Hammurabi to Legendre, Birkhaüser, Basel, 1984. 